\title{
Comparison of aerodynamic characteristics provided by wing with bell shaped lift distribution and generalized wings
}

\author{
Pavel Hospodá: ${ }^{1,},{ }^{*}$, Armand Drábek $^{2}$ \\ ${ }^{1}$ VZLU, Aerodynamic Department, 19905, Beranových 130, Prague Czech Republic
}

\begin{abstract}
In this article different wings are computed by low and highfidelity methods to compare their aerodynamic characteristics. Thanks to the unusual properties of the wing with the bell-shaped lift distribution, several general geometrical variants of the wings were calculated and their results are presented in this work. Three general wings are assumed and their geometry is defined as rectangular, trapezoidal and elliptical. Airspeed, total lift force, shape of airfoil and root chord are defined, and bending moment is assumed as a surrogate model for wing weight. The goal of optimization is minimization of aerodynamic drag.
\end{abstract}

\section{Introduction}

Ludwig Prandlt developed computational method to calculate elementary characteristics of finite span wing at the beginning of aviation. He also derived wing with best aerodynamic efficiency as a wing with elliptical list distribution [1]. Several years later, he conceded the previous conclusion leads to an invalid result, there was a superior spanload solution that maximizes efficiency for a given bending moment [2] His new bell-shaped spanload creates a wing that is 11 percent more efficient and has 22 percent greater span. This solution is called Bell-shaped lift distribution (BSLD) and is depicted in Figure 1. This knowledge remains unfortunately unknown.

Sometimes around 1935 Reimar Horten independently derived an approximate equivalent to Prandtl's second solution. Horten, on the other hand, did calculate the induced drag across the span of the wing, and in 1950 concluded that something singularly possible existed, but he never explained.

Robert Jones optimized a lift distribution for a given lift and bending moment in 1950 [3]. His result is close to BSLD and has $15 \%$ higher efficienty and $15 \%$ grater wings related to elliptical spanload.

Armin Klein and S. P. Viswanathan developed similar solution in his work [4],[5]in the 1973 and 1975 respectively.

And finally, Albion Bowers published: On wings of the minimum induced drag - which translation of Prandl's second paper [6]. He completely rediscovered BSLD, its consequence and made flight test to proof of concept.

\footnotetext{
* Corresponding author: hospodar@,vzlu.org
} 


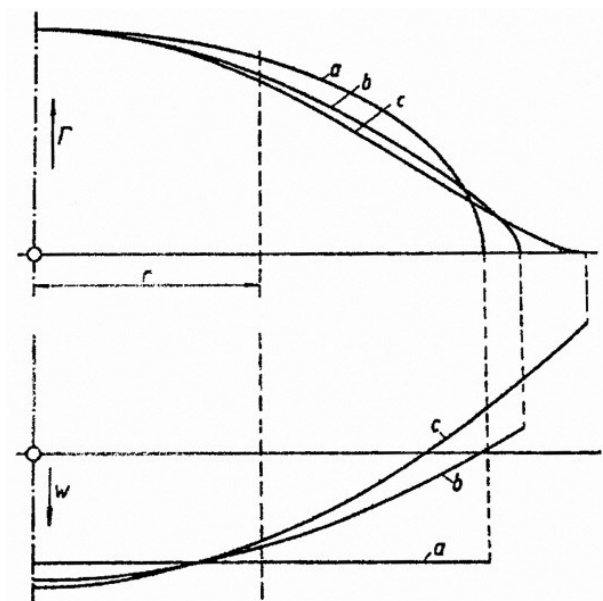

Fig. 1. Comparison of wings with different distribution of circulation and downwash velocity (line "a" elliptical distribution, line "c" bell shaped lift distribution $)^{\dagger}$

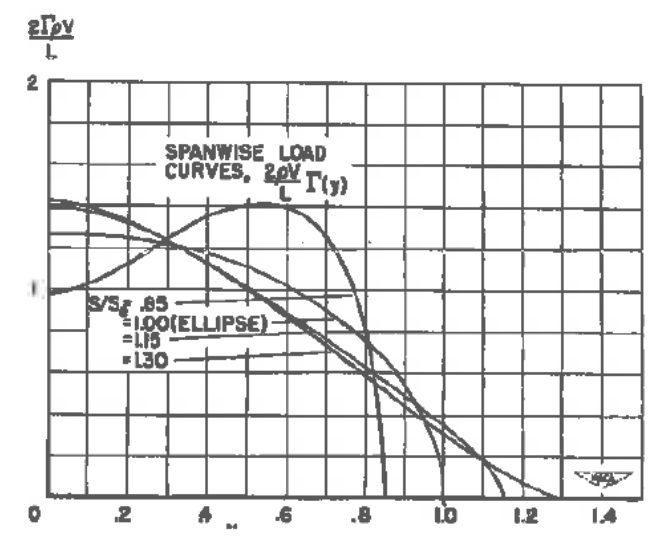

Fig. 2. Different spanload distribution calculated by Jones $\$$,as a base line a wing with elliptical lift distribution is used

\section{Computational procedure}

In this work a 4 different wing geometry was optimized and analyzed. For that reason, a low and high fidelity computational methods were used. As a computational solver for optimization a Lifting line theory is used. Response surface method procedure was chosen to find out a global optimum. After optimization a CFD calculation was done for comparison of different computational method.

\subsection{Lifting line theory}

Ludwing Prandtl explained aerodynamic forces and moments in the lifting line theory [1] A finite span wing is assumed in this paper and moreover, there was derived an optimal lift distribution produced minimum induced drag. A solution is elliptical lift distribution. Prandtl developed a vortex scheme where each vortex induces downwash velocity. Basic characteristic in LLT is downwash velocity $w$ and it can be computed in the side position $y_{0}$ :

$$
w\left(y_{0}\right)=\frac{1}{4 \pi} \int_{-b / 2}^{b / 2} \frac{d \Gamma}{y-y_{0}}
$$

Circulation is a function of lift coefficient and this depends on the induced angle of attack and downwash velocity respectively. Thus, this equation is in implicit form and has to be solved numerically. Although lifting line theory is based on a potential flow without viscosity effect, a viscous drag can be estimated through two-dimensional airfoil data. Therefore, a local lift coefficient $c_{l}$ is interpolated from nonlinear aerodynamic characteristic based on the total angle of attack $\alpha$. Lift coefficient and local chord $c$ and is computed by following terms:

$$
l=\frac{1}{2} \rho \cdot V^{2} \cdot c_{l}(\alpha) \cdot c
$$

\footnotetext{
$\dagger$ Taken over reference [2]

$\$$ Taken over reference [3]
} 
Then a Kutta-Joukovsky law is used to determine local circulation $\Gamma$. This concept was verified in previous work [7].

\subsection{CFD}

Models of different wing platforms were created in CATIA program. Meshing procedure was made in POINTWISE consequently. Meshes had about 40 million of elements with hexahedral grid on surface. Volumes were filled by tetrahedral elements. The huge amount of elements was placed in density region to catch vortex structures. Boundary layer was simulated by 60 prismatic layers.

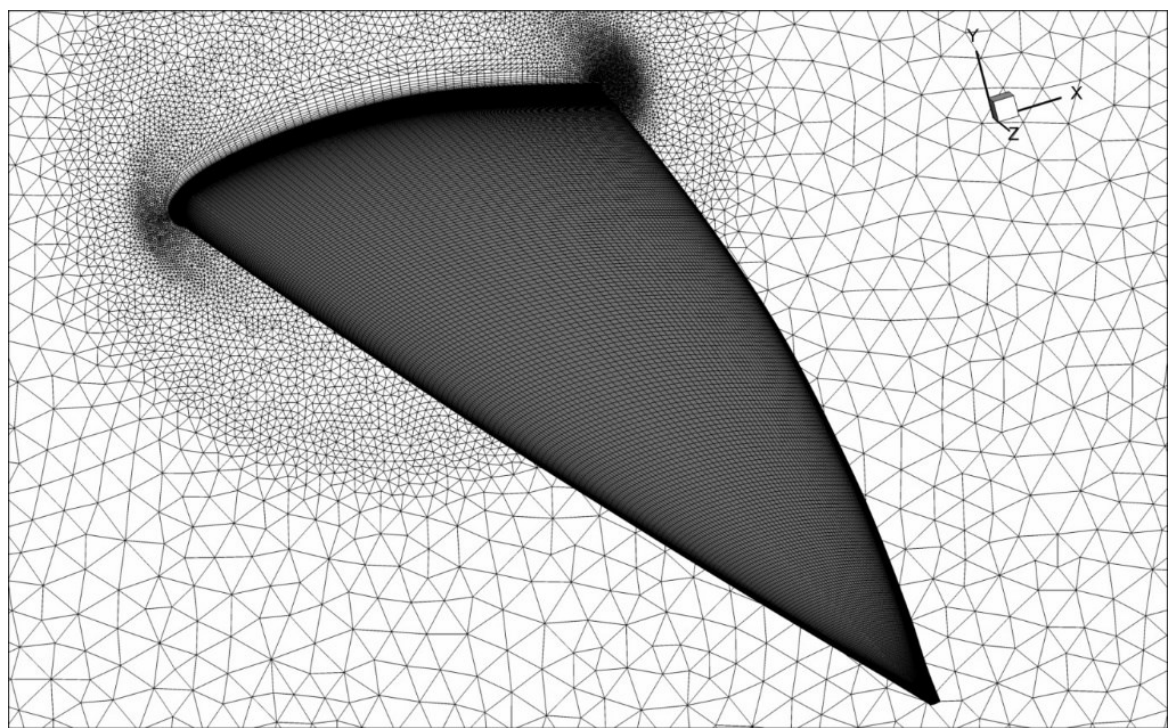

Fig. 3. Mesh over the optimized wing. Picture depicts the surface grid. On the symmetry plane can be seen the prismatic layers. Smoothed grid can be seen in the density region. The surface of the wing is meshed with hexahedral elements.

For computations Edge program was chosen. Solver is suitable for compressible, viscous/inviscid flow. All computations were made with Navier stokes averaged equations with W\&J EARSM + Hellsten k-omega Turbulence model and Central scheme discretization. The main objective of this analysis was the comparison of aerodynamic characteristics with low fidelity methods. Graphical output was made in TECPLOT to see the flow field over the computed wing.

\subsection{Optimization of wings geometry}

There is a description of optimization procedure. Cost function, that has to be minimized, is total aerodynamic drag as a function of design parameter. Bending moment is assumed surrogate for wing weight. Lift is prescribed and determined from polar for specified angle of attack. Geometry of different wing is based on design vector defined in the table 1 . Three general wing geometries are defined using chord (root/tip) and wing span. Wing with BSLD has moreover specified chord distribution around wing span. The chord distribution is optimized in the inner loop to reach a bell shaped lift distribution. For that reason, a gradient method is used to inner optimization. 
Table 1. design parameters for different wing geometry

\begin{tabular}{|c|c|}
\hline Wing shape & Design parameters \\
\hline Rectangular & Chord, wing span \\
\hline Elliptical & Chord, wing span \\
\hline Trapezoidal & Root and tip chord, wing span \\
\hline BSLD & Root chord, wing span \\
\hline
\end{tabular}

As a design procedure a Response surface methodology (RSM) is used [8] in this work. It is simple and relatively widely used methodology in optimization problems [9]. This kind of methodology is based on least squares method and is used as surrogate model for description of design space. In the first step of optimization a few points are calculated to estimate a shape of design space. Then more points in local optimum are counted and further selected for Response surface methodology. After that a RSM is used to find out a local optimum. This approach is used for all wing geometries. Lifting line theory is used for optimization procedure and after reaching of optimum a CFD is used to validate a design. Optimal shapes of the wing geometry are depicted with pressure distribution in the following figure.

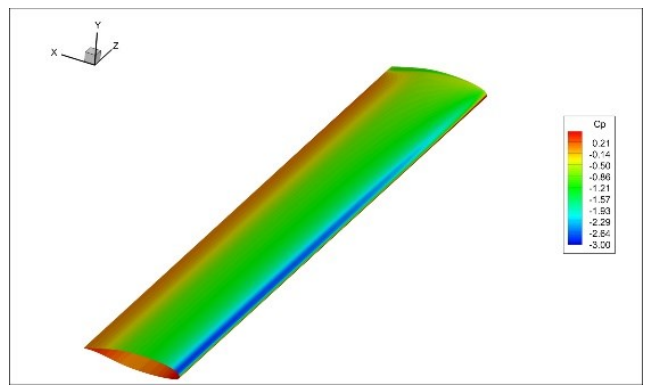

(a)

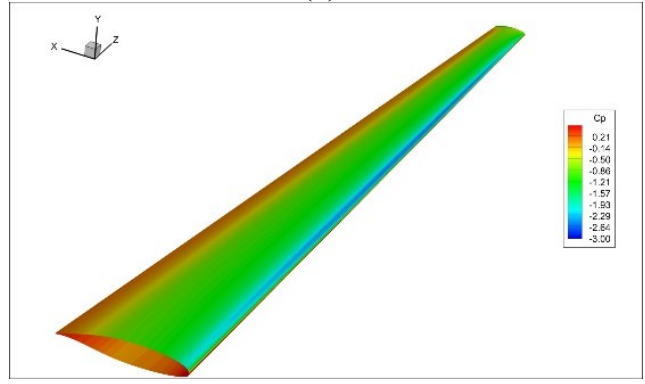

(c)

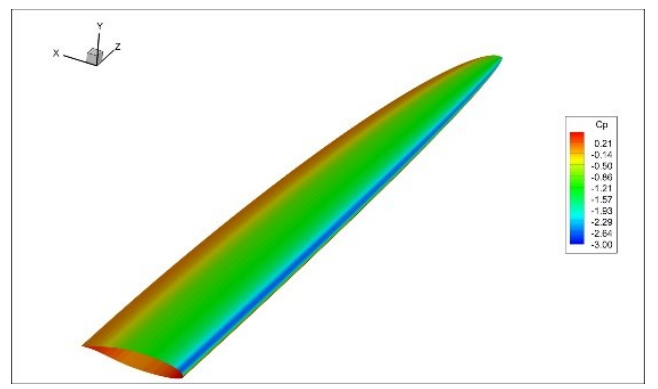

(b)

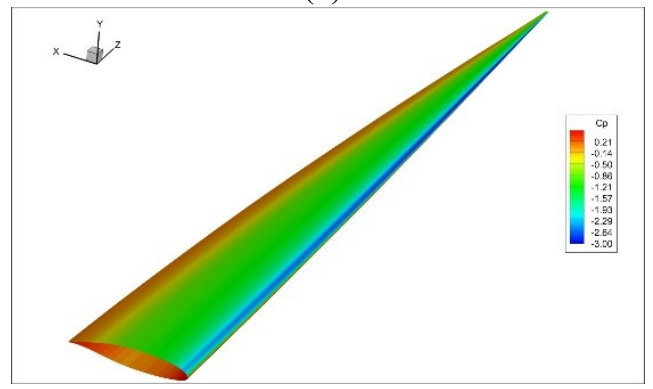

(d)

Fig. 4. Comparison of wings geometry and pressure distribution; (a) - rectangular wing; (b) elliptical; (3) - trapezoidal wing; (4) - wing with bell-shaped lift distributio 


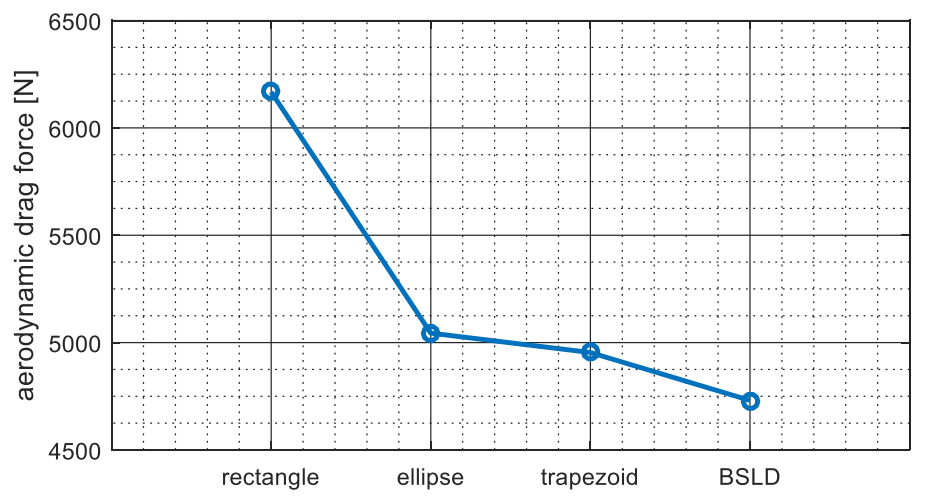

Fig. 3. Aerodynamic drag force for different optimal wing geometry

\section{Conclusion}

The smallest wingspan has for the rectangular wing and it has the highest aerodynamic drag (see fig. 5). Elliptical and trapezoidal wings have similar values of aerodynamic drag. The trapezoidal wing has lower drag than elliptical but it has also a little bit larger wingspan. Wing geometry with bell-shaped lift distribution has the lowest aerodynamic drag for given lift and bending moment. It has $8 \%$ lower aerodynamic drag and $14 \%$ larger wing span related to elliptical wing.

\section{Aknowlegement}

This result was achieved within the institutional support of the Ministry of industry and trade for the development of the research organization.

\section{References}

1. Prandtl, L.: Tragflügeltheorie, Königliche Gesellschaft der Wissenschaften zu Göttingen (1918)

2. Prandtl L.: Über tragflügel kleinsten induzierten widerstandes. Zeitschrift für Flugtecknik und Motorluftschiffahrt, München, Deustchland, 1 VI (1933)

3. Jones T. R.: The spanwise distribution of lift for minimum induced drag of wings having a given lift and a given bending moment, Document ID: 19930082889, NACA Technical Note 2249, Dec 01, (1950)

4. Klein, A., Viswanathan, S. P. (1973). Minimum induced drag of wings with given lift and root-bending moment. Zeitschrift Für Angewandte Mathematik Und Physik ZAMP, 24(6), 886-892. doi:10.1007/bf01590797

5. Klein, A., Viswanathan, S. P. (1975). Approximate solution of minimum induced drag of wings with given structural weight. Journal of Aircraft, 12(2), 124-126. doi: $10.2514 / 3.44425$

6. Bowers A. H., Murillo O. J., Jensen R., Eslinger B., Gelzer Ch.: On Wings of the Minimum Induced Drag: Spanload Implications for Aircraft and Birds ,Technical Report Document ID: 20160003578, NASA Armstrong Flight Research Center; Edwards, CA, United States, Mar 01, 2016 
7. Hospodář P., Klesa J., Žižkovský N.: Wing and propeller aerodynamic interaction through nonlinear lifting line theory and blade element momentum theory. MATEC Web of Conferences, 233, 00027. doi:10.1051/matecconf/201823300027, (2018)

8. Vrchota P., Hospodář P., Response Surface Method Application to High-Lift Configuration with Active Flow Control, Journal of Aircraft, Vol. 49, No. 6, pp. 17961802. 2012

9 Raymond H. Myers, Douglas C. Montgomery, Christine M. Anderson-Cook.: Response Surface Methodology: Process and Product Optimization Using Designed Experiments, 3rd Edition. John Wiley \& Sons. ISBN: 978-0-470-17446-3, 2009 